\title{
Carbon Dioxide Angiography
}

\section{Brian Funaki, M.D. ${ }^{1}$}

The algorithm for patients with renal insufficiency who require vascular imaging has recently changed in my hospital. Previously, the majority of these patients underwent magnetic resonance angiography (MRA) or occasionally computed tomographic angiography after prescan hydration and sodium bicarbonate infusion. Although we have used carbon dioxide $\left(\mathrm{CO}_{2}\right)$ for many years, with the advent of nephrogenic systemic sclerosis and its unclear relationship to MRA contrast agents, we are increasingly using $\mathrm{CO}_{2}$ as a contrast agent for both diagnosis and to guide therapy. $\mathrm{CO}_{2}$ has been used for $>30$ years. It is not nephrotoxic or hepatotoxic and is nonallergenic. It is also quite inexpensive compared with other contrast agents.

\section{PROCEDURE}

\section{Equipment}

I use a modified plastic bag system with an O-ring first described by Hawkins and associates. A prepackaged carbon dioxide kit (called a "custom waste management kit" from Merit Medical, South Jordan, UT) is available. A similar kit is manufactured by AngioDynamics (Queensbury, NY). Because $\mathrm{CO}_{2}$ angiography is "offlabel," neither can be marketed as a " $\mathrm{CO}_{2}$ angiography" kit, and both cost approximately $\$ 50$ to 100 . Although very straightforward, it is important to understand the connections of this system thoroughly (Fig. 1). The kit includes the following:

- $1500-\mathrm{mL} \mathrm{CO} 2$ bag

- 50-mL syringe

- Multiple stopcocks and connectors

- Medical-grade $\mathrm{CO}_{2}$ cylinder

\section{Filling Reservoir Bag}

The bag is purged three times using an O-ring filter with medical grade $\mathrm{CO}_{2}$ to eliminate room air. On the final filling, the bag should not be overdistended.

\section{Connection to Catheter}

The filled bag is connected to the remaining manifold and the two-way stopcock is opened. The $50-\mathrm{mL}$ syringe is filled with $\mathrm{CO}_{2}$ via a dual check valve and expelled to purge air out of the tubing, which is then connected to the angiographic catheter. The three-way stopcock can be turned off to $\mathrm{CO}_{2}$ to allow backbleeding into a small syringe to eliminate all air from the system. The stopcock is then closed to the backbleeding syringe, and the blood is gently purged from the catheter by slowly injecting $\mathrm{CO}_{2}$ via the syringe. When injecting $\mathrm{CO}_{2}$, resistance is initially felt as blood is purged from the catheter followed by a "give" when $\mathrm{CO}_{2}$ completely fills the catheter. Blood is purged from the catheter to prevent "explosive" delivery of $\mathrm{CO}_{2}$ during angiography.

\section{Angiography}

Angiographic runs are then performed at two to six frames per second using digital subtraction software with angiographic inversion and image summation, or "stacking." Typically 30 to $50 \mathrm{~mL}$ of $\mathrm{CO}_{2}$ are injected into the aorta or inferior vena cava via a pigtail catheter. Less volume is used for selective injections, and it is helpful to use catheters with one side hole. Injections are performed 2 to 3 minutes apart; occasionally slightly longer delays are used when performing mesenteric angiography. Patients should be supine, and injections must be made below the level of the diaphragm. For evaluation of the calves and feet, the legs can be elevated slightly on pillows to allow the gas to "rise" to fill the arteries in this region. Nitroglycerine in $100-$ to $200-\mu \mathrm{g}$ aliquots can be administered intra-arterially as necessary.

\section{Interpretation}

It is important to recognize that $\mathrm{CO}_{2}$ "floats" on the top of blood; therefore abnormalities in the dependent portions of blood vessels (e.g., posterior plaques in iliac arteries) may not be demonstrated to full advantage. In practical terms, this is only a problem in larger (i.e., $>1-\mathrm{cm}$

\footnotetext{
${ }^{1}$ Section of Vascular and Interventional Radiology, University of Chicago Medical Center, Chicago, Illinois.

Address for correspondence and reprint requests: Brian Funaki, M.D., Section of Vascular and Interventional Radiology, University of Chicago Medical Center, 5840 S. Maryland Avenue, MC 2026, Chicago, IL 60637.
}

New Tools and Techniques in Interventional Radiology; Guest Editor, Martin G. Radvany, M.D.

Semin Intervent Radiol 2008;25:65-70. Copyright (C) 2008 by Thieme Medical Publishers, Inc., 333 Seventh Avenue, New York, NY 10001, USA. Tel: +1(212) 584-4662.

DOI 10.1055/s-2008-1052308. ISSN 0739-9529. 
A
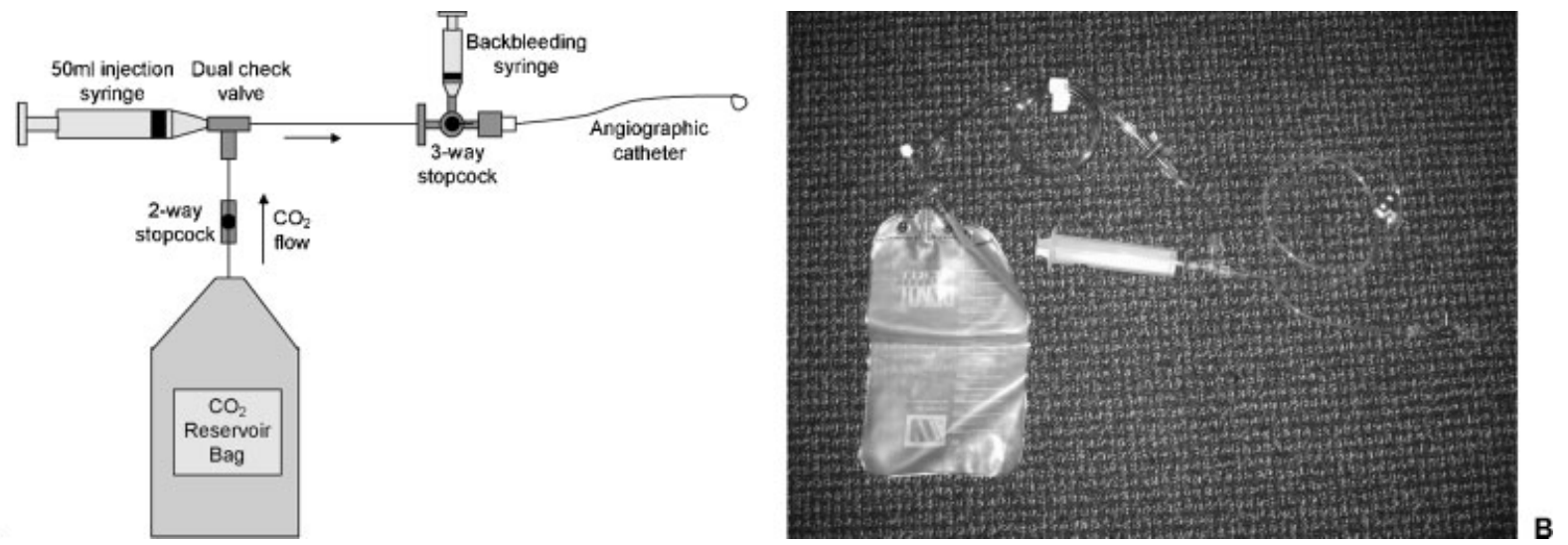

Figure 1 Carbon dioxide delivery system. (A) Illustration depicts connections in closed bag system. (B) Photograph of closed bag system.

diameter) vessels because $\mathrm{CO}_{2}$ fills smaller caliber arteries more completely. Arteries that assume a posterior course, such as dependent coursing renal arteries, may be difficult to fill, particularly from a nonselective injection. $\mathrm{As}_{\mathrm{s}} \mathrm{CO}_{2}$ passes through bifurcations, the bolus dissipates into smaller bubbles that can create a "pseudostenosis" (Fig. 2). Overlying bowel gas can obscure vessels and presents a potential pitfall to interpretation (Fig. 3).

\section{Complications and Contraindications}

The following complications have been reported using $\mathrm{CO}_{2}$ for angiography: paresthesia, abdominal pain, tenesmus, vapor lock, and nausea. Abdominal pain is thought to be related to $\mathrm{CO}_{2}$ in the mesenteric vessels and is best handled by rotating the patient from side to side and gently massaging the abdomen to allow the $\mathrm{CO}_{2}$ to diffuse into the blood. It may be caused by vapor lock in mesenteric arteries and is reported to be more likely in patients with $\mathrm{CO}_{2}$ collecting in abdominal aortic aneurysms. Nausea is usually encountered only when high flow rates are used for angiography.

Vapor lock in the heart is a feared complication that occurs when $\mathrm{CO}_{2}$ is trapped there, increasing pulmonary artery pressure and preventing normal blood flow and venous return. This is essentially only a risk with venography and may reflect air contamination. Intravascular air injection may cause significant vapor lock in the heart with bradycardia and hypotension. If this occurs, the patient should be rotated into a left lateral decubitus position.

$\mathrm{CO}_{2}$ should not be combined with nitrous oxide sedation, and it should be used with discretion in patients with chronic obstructive pulmonary disease. $\mathrm{CO}_{2}$ is contraindicated in the cerebral vessels and should

\section{A}
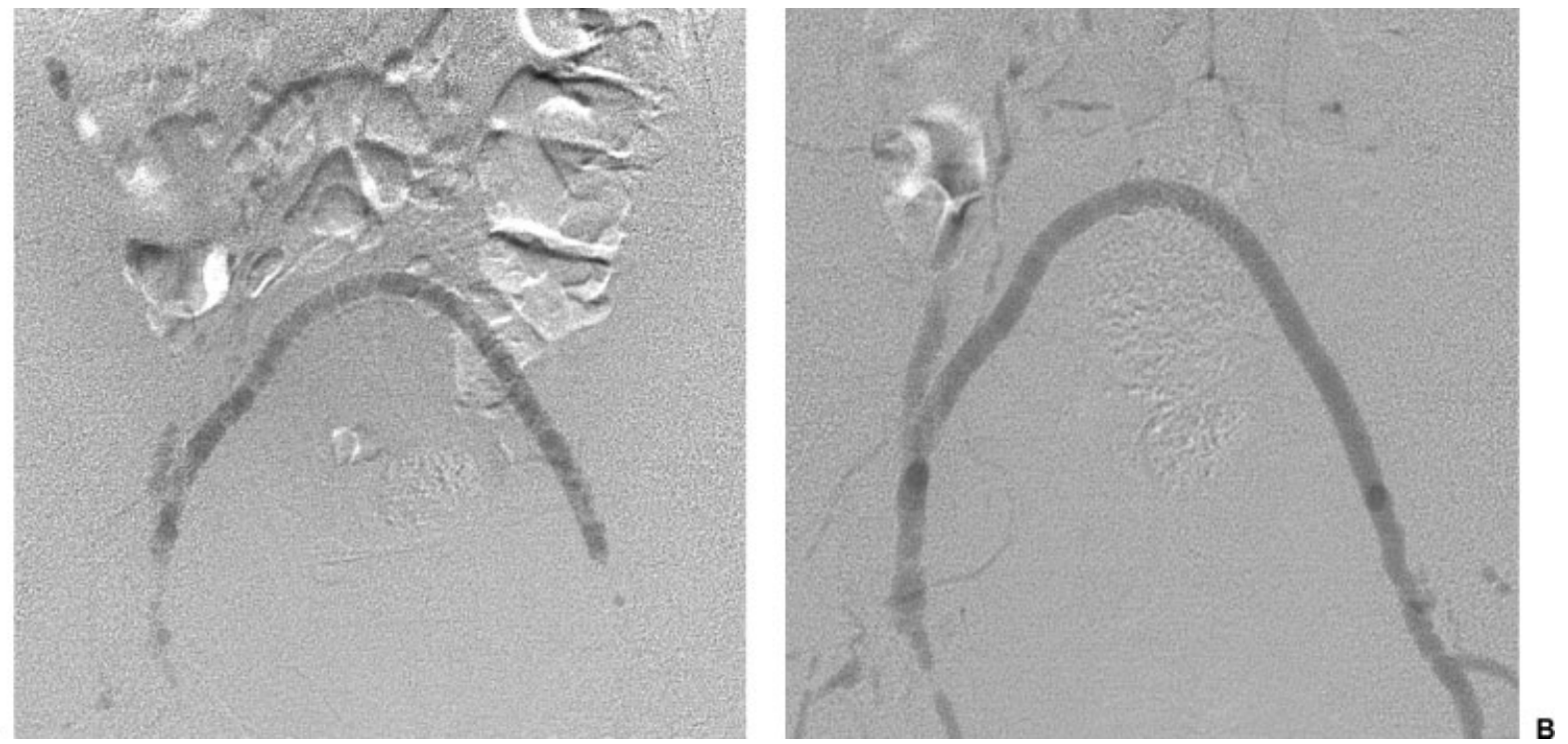

Figure 2 Carbon dioxide $\left(\mathrm{CO}_{2}\right)$ pelvic angiography in a patient with a femoral-to-femoral crossover graft. (A) Digital subtraction angiogram with insufficient $\mathrm{CO}_{2}$ injected, resulting in poor filling of graft. (B) Repeat digital subtraction angiogram with larger bolus showing widely patent graft. 

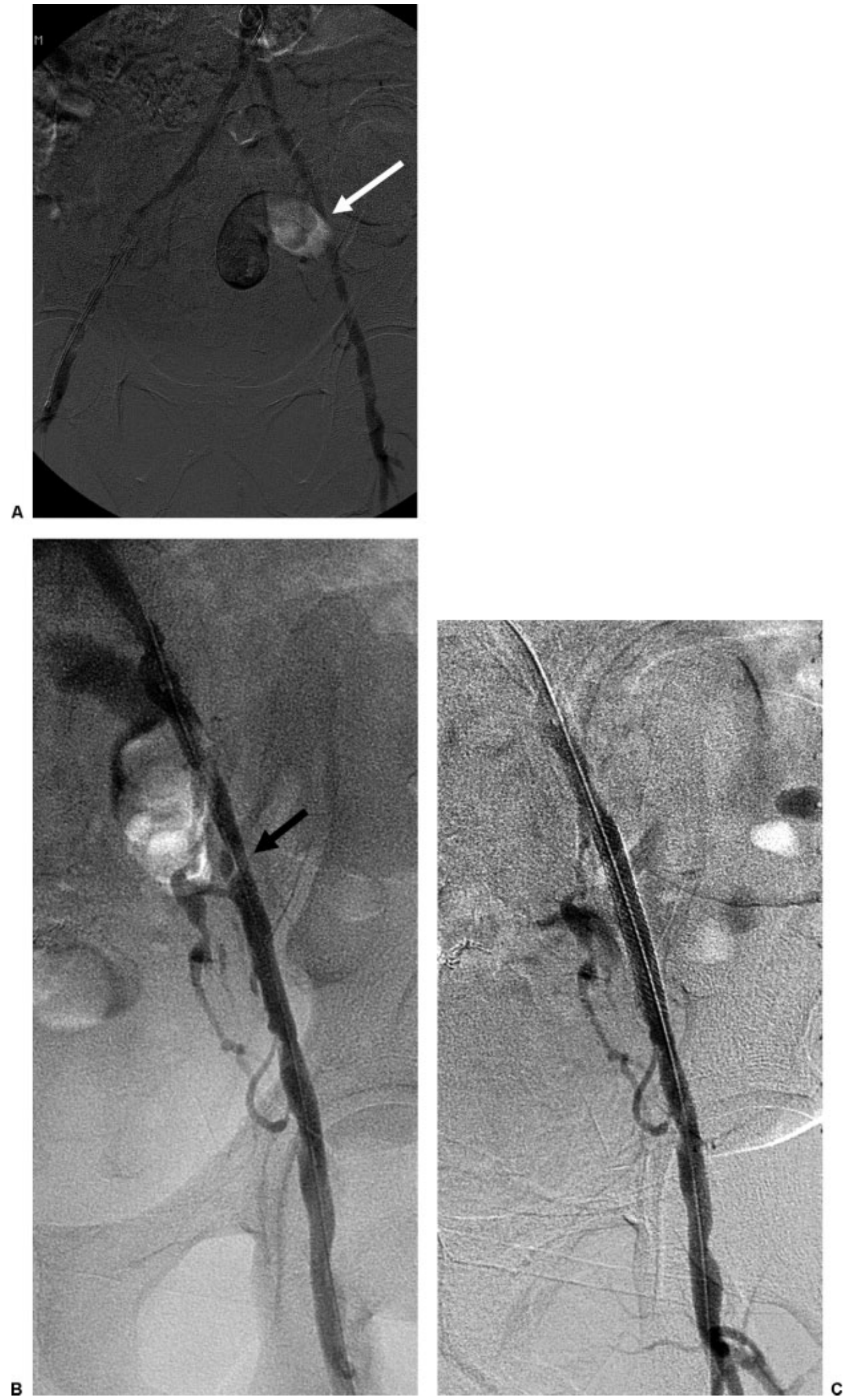

Figure 3 Bowel gas pitfall. (A) Digital subtraction angiogram using carbon dioxide in which bowel gas partially obscures left external iliac artery (white arrow). (B) Digital subtraction angiogram using iodinated contrast material shows ulcerated plaque and high-grade stenosis (black arrow). (C) Digital subtraction angiogram after stenting shows alleviation of lesion. 
A
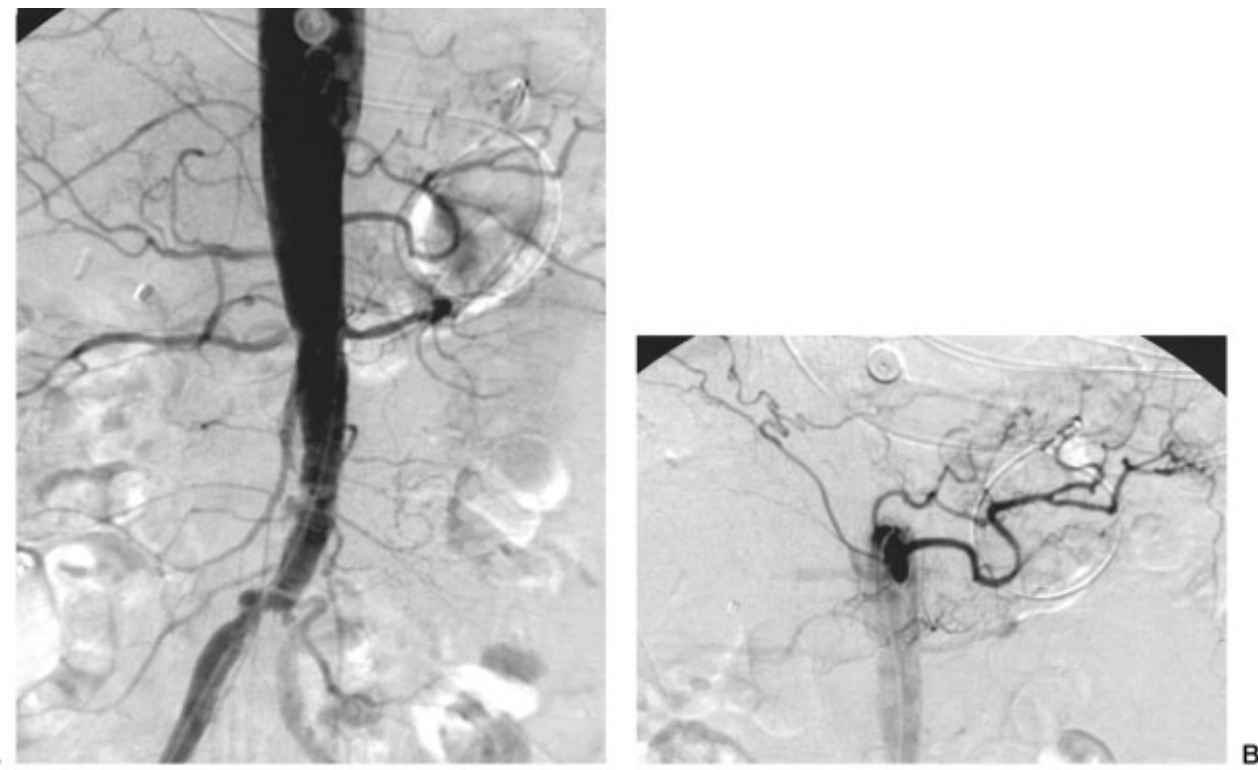

C
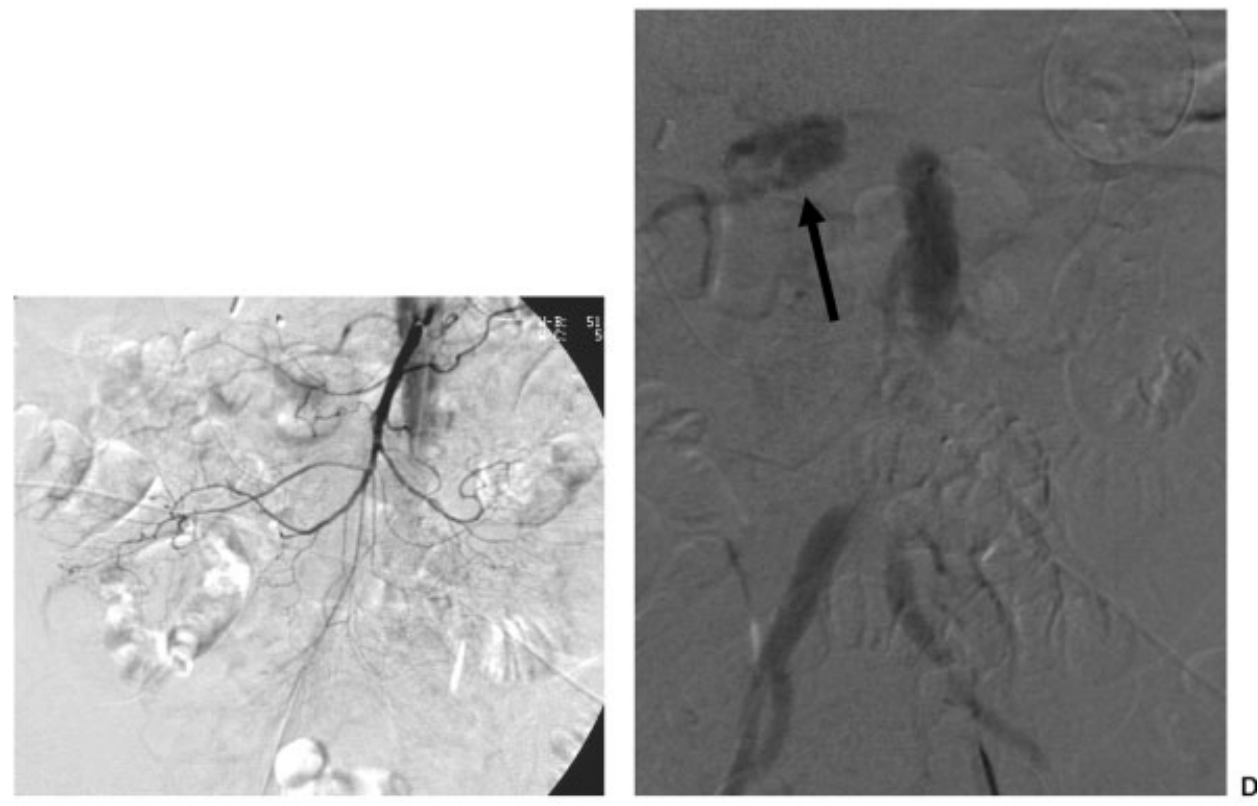

E

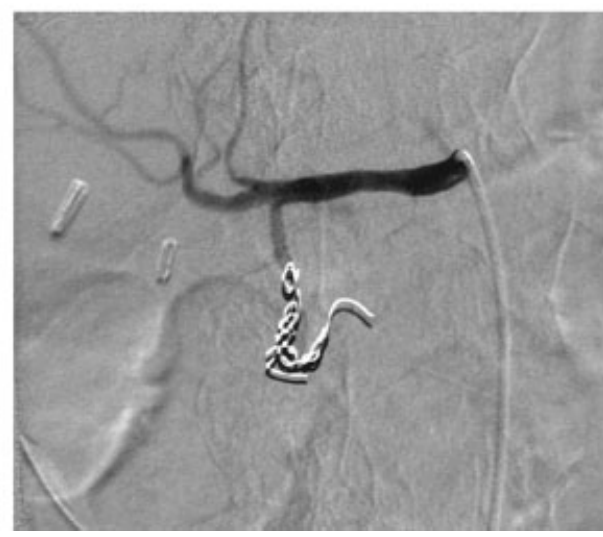

Figure 4 Carbon dioxide $\left(\mathrm{CO}_{2}\right)$ for gastrointestinal bleeding from obscure source. Tagged red blood cell scintigraphy showed bleeding in the right upper quadrant. (A) Aortogram using iodinated contrast material fails to show bleeding. (B) Celiac arteriogram fails to demonstrate bleeding. (C) Superior mesenteric angiogram fails to show bleeding. (D) $\mathrm{CO}_{2}$ aortogram showing bleeding in the right upper quadrant (arrow). (E) Digital subtraction angiogram after embolization. Active bleeding was found from a replaced gastroduodenal artery arising directly from the aorta, which was then embolized. 


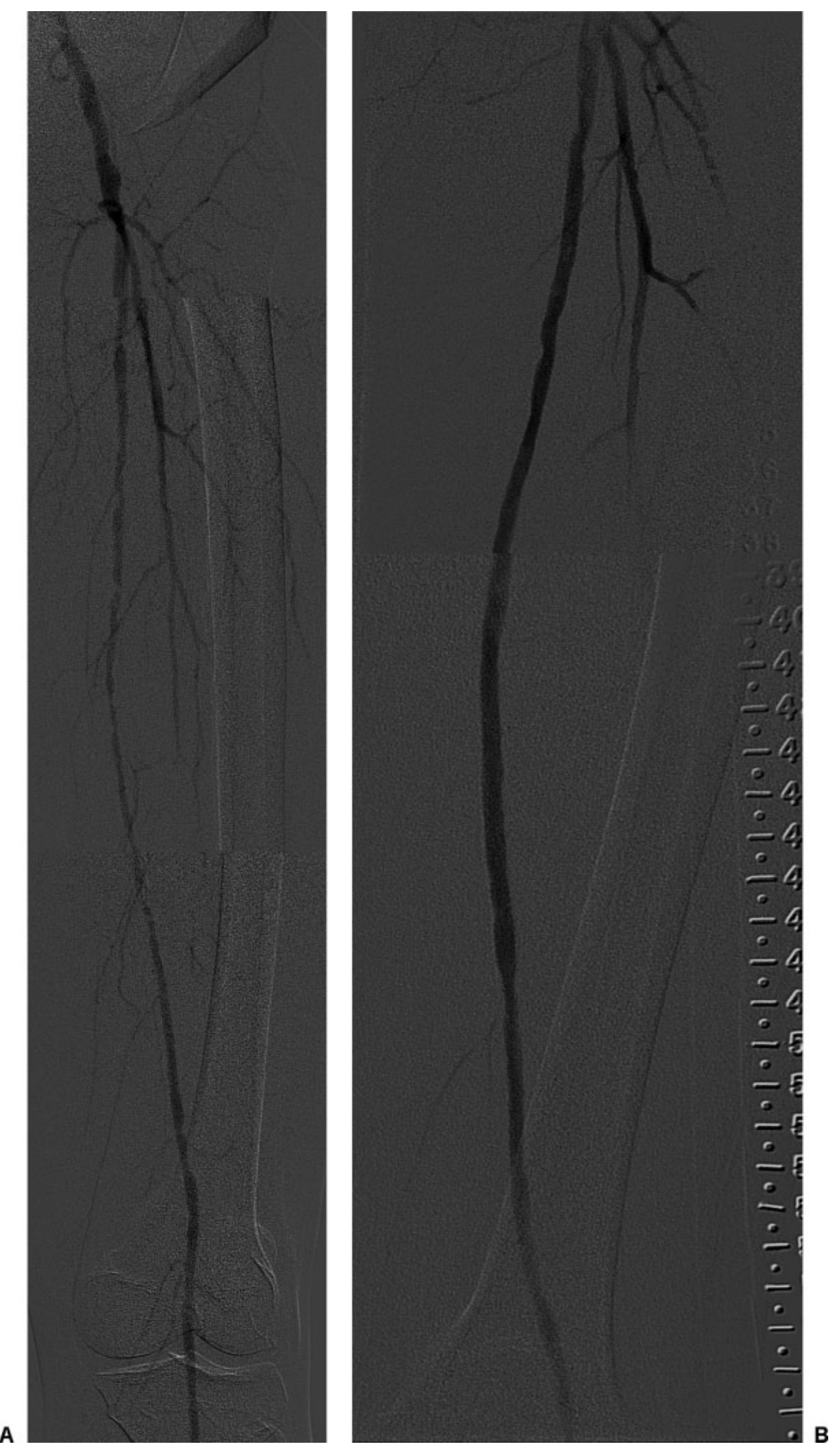

Figure 5 Superficial femoral artery intervention. (A) Composite digital subtraction angiogram using carbon dioxide shows diffuse atherosclerotic disease of superficial femoral artery. (B) Composite digital subtraction angiogram after covered stent placement shows widely patent artery. 
not be injected above the level of the diaphragm or used in patients with left-to-right shunts.

\section{DISCUSSION}

Hawkins, Caridi, and associates at the University of Florida have accrued the longest and largest experience with $\mathrm{CO}_{2}$, which can be used for a variety of procedures:

- Transjugular intrahepatic portosystemic shunt (TIPS) insertion: $\mathrm{CO}_{2}$ is used to perform wedged portal venography; however, there have been several case reports of hepatic capsular rupture and death when using $\mathrm{CO}_{2}$ for this purpose. Personally, I do not routinely perform wedged venography during TIPS. I do not find it particularly helpful. If $\mathrm{CO}_{2}$ is used for this purpose, some investigators advocate gentle injection via an occlusion balloon inflated in the hepatic vein.

- Gastrointestinal (GI) hemorrhage: $\mathrm{CO}_{2}$ is much less viscous compared with liquid contrast material and therefore may be more sensitive for the detection of GI bleeding. I have found it helpful in problematic cases (Fig. 4) but do not perform it routinely for all procedures.

- Diagnostic angiography and intervention: I routinely use $\mathrm{CO}_{2}$ for diagnostic angiography in patients with renal insufficiency. In most cases, it can be used for both diagnosis and intervention (Fig. 5). Typically, I augment the study with a small volume of iso-osmolar contrast (10 to $20 \mathrm{~mL}$ ) to show any pathology to best advantage or clarify any equivocal findings. I dilute the contrast 1:1 and use it for selective or subselective injections.
- Renal intervention: Similar to diagnostic angiography, I often use $\mathrm{CO}_{2}$ for renal interventions. Occasionally, it is helpful to rotate the patient slightly to help fill the artery. Again, due to its relatively low viscosity, it is much easier to inject $\mathrm{CO}_{2}$ around a guidewire via a sidearm adaptor compared with liquid contrast. If necessary, I augment the study with small volumes of iso-osmolar contrast.

- Venography: Can be done prior to inferior vena cava filter placement. It is more difficult to visualize posterior oriented renal veins (i.e., right renal vein) and caval thrombus compared with liquid contrast. $\mathrm{CO}_{2}$ can also be used for arm venography prior to dialysis access creation for patients with renal insufficiency who have not completely lost renal function or in patients with allergies to iodinated contrast material. In all these interventions, it is important to check the pulmonary arteries for accumulation of gas and subsequent dissipation within 30 to 45 seconds.

\section{SUGGESTED READINGS}

Hawkins IF, Caridi JG. Carbon dioxide $\left(\mathrm{CO}_{2}\right)$ digital subtraction angiography: 26-year experience at the University of Florida. Eur Radiol 1998;8:391-402

Hawkins IF, Caridi JG, Klioze SD, Mladinich CR. Modified plastic bag system with O-ring fitting connection for carbon dioxide angiography. AJR Am J Roentgenol 2001;176:229232

Shaw DR, Kessel DO. The current status of the use of carbon dioxide in diagnostic and interventional angiographic procedures. Cardiovasc Intervent Radiol 2006;29:323-331 